\title{
A CURVILINEAR EXTENSION OF IVERSEN-TSUJI'S THEOREM FOR SIMPLY CONNECTED DOMAIN
}

\author{
UN HAING CHOI
}

\begin{abstract}
Let $D$ be a simply connected domain with at least two boundary points in the complex plane, and $t$ a boundary point of $D$. For a meromorphic function $f(z)$ in $D, \lim \sup |f(z)|$ as $z \rightarrow t$ is given in terms of accessible boundary points and prime ends. This gives a curvilinear extension of Iversen-Tsuji's Theorem for a simply connected domain.
\end{abstract}

1. Introduction. Let $D$ be an arbitrary domain in the complex domain, $\partial D$ its boundary, $E$ a compact subset of capacity zero on $\partial D$, and let $t_{0}$ be a point of $E$ such that $N\left(t_{0}\right) \cap(\partial D-E) \neq \varnothing$ for every neighborhood $N\left(t_{0}\right)$ of $t_{0}$.

Iversen-Tsuji's Theorem [1, p. 16] states that if $f(z)$ is a meromorphic function in $D$ and bounded in the intersection of $D$ with some neighborhood of $t_{0}$, then

$$
\limsup _{z \rightarrow t_{0}, z \in D}|f(z)|=\limsup _{b \rightarrow t_{0}, b \in \partial D}\left(\limsup _{z \rightarrow b}|f(z)|\right) .
$$

We give a curvilinear extension of this theorem for an arbitrary simply connected domain.

2. Definitions. A subset of the boundary of a simply connected domain $D$ with at least two boundary points will be called a $D$-conformal null set if it corresponds to a set of linear measure zero under a one-to-one conformal mapping onto the unit disc. The set of all prime ends of $D$ will be denoted by $\tilde{D}$. If @ is an accessible boundary point of $D$, then@ determines a unique prime end $P(@)$.The complex coordinate of an accessible boundary point @ will be denoted by $z(@)$.

Now we are ready to state our result.

3. THEOREM. Let $D$ be a simply connected domain in the complex plane, which is not the whole plane, $t_{0}$ a boundary point of $D, \tilde{E}$ a conformal null set of prime ends of $D$. If $f(z)$ is meromorphic in $D$ and bounded in the intersection of $D$ with some neighborhood of $t_{0}$, then

$$
\limsup _{z \rightarrow t_{0}, z \in D}|f(z)|=\limsup _{z(@) \rightarrow t_{0}, P(@) \in \tilde{D}-\tilde{E}}\left(\inf _{A}\left(\limsup _{z \rightarrow z(@), z \in A}|f(z)|\right)\right),
$$

where $A$ is an arc at an accessible boundary point @with $P(@) \in \tilde{D}-\tilde{E}$ and the convergence is in the sense of the ordinary euclidean metric.

Proof. We may assume that $|f|$ is bounded by 1 (by multiplying a suitable

Received by the editors October 17, 1975.

AMS (MOS) subject classifications (1970). Primary 30A72; Secondary 30A42.

Key words and phrases. Capacity, conformal null set, prime end, accessible boundary point, Perron process, Poisson integral, ambiguous point, $\frac{1}{2}$-dimensional Hausdorff measure. 
constant). Let $m=$ the right-hand side of (1). Then it suffices to show that $\lim \sup _{z \rightarrow t_{0}, z \in D}|f(z)| \leqslant m$.

Case $1 . m>0$. Suppose the statement is not true. Then there exists a sequence $\left\{z_{n}\right\}$ in $D$ converging to $t_{0}$ such that $\left|f\left(z_{n}\right)\right|$ converges to a positive constant $c$ greater than $m$. Choose $N$ so that $\left|f\left(z_{n}\right)\right|>m+(c-m) / 2$ for all $n>N$.

Let $\varepsilon$ be an arbitrary positive number less than $(c-m) / 8$. Choose $r>0$ so that at each accessible boundary point@with $P(@) \in \tilde{D}-\tilde{E}$ and its complex coordinate in $\bar{D}_{r} \cap \partial D\left(\bar{D}_{r}\right.$ is the closure of $D_{r}=\left\{\left|z-t_{0}\right|<r\right\}$, and $\partial D$ is the boundary of $D$ ), there exists an arc on which $|f(z)|<m+\varepsilon$.

Let $G_{n}$ be the component of $D \cap D_{r}$ containing $z_{n}(n>N)$.

Let $G_{0}$ be the domain obtained by adding the ring domain $\{z: r / 2<$ $\left.\left|z-t_{0}\right|<2 r / 3\right\}$ to the union of the $G_{n}$ intersecting the ring domain (if $D \cap D_{r}$ has just one component, let $G_{0}$ be the component).

Let $\phi(t)$ be the real-valued function defined on $\partial G_{0}$ in the following way:

$$
\begin{aligned}
\phi(t) & =0 \text { for } t \in \partial G_{0} \cap\left\{z:\left|z-t_{0}\right|<r / 2\right\}, \\
& =\log (2 / m) \quad \text { elsewhere. }
\end{aligned}
$$

Let $u(z)$ be the harmonic function obtained from the Perron process with $\phi(t)$ and $G_{0}$. Let $g_{n}(w)$ be a one-to-one conformal mapping from the unit disc $D_{w}$ onto $G_{n}$. Note that $f\left(g_{n}(w)\right)$ is a bounded analytic function in $D_{w}$. Using Theorem 2.9 in [2, p. 30], $f\left(g_{n}(w)\right)$ can be written as

$$
f\left(g_{n}(w)\right)=w^{m} B(w) \exp (h(w)),
$$

where $h(w)$ is an analytic function with negative real part in $D_{w}$, and $B(w)$ is the Blaschke product of zeros of $f\left(g_{n}(w)\right)$. For each $n$ we write

$$
f\left(g_{n}(w)\right)=B_{n}(w) \exp \left(h_{n}(w)\right) .
$$

If $f\left(g_{n}(w)\right)$ has no zero in $D_{w}$, let $B_{n}(w) \equiv 1$. Then the function $f\left(g_{n}(w)\right) / B_{n}(w)$ is a bounded analytic function with no zero in $D_{w}$, and $\log \left|f\left(g_{n}(w)\right) / B_{n}(w)\right|$ is a negative harmonic function. We note that $B_{n}(w)$ has a radial limit with modulus 1 almost everywhere on $\partial D_{w}[2$, Theorem 2.11 , p. 32].

We consider the harmonic function

$$
v(w)=\log \left|f\left(g_{n}(w)\right) / B_{n}(w)\right|-u\left(g_{n}(w)\right), \quad w \in D_{w} .
$$

We know that for almost every $e^{i \theta}$ on $\partial D_{w}$, the image of the radius $t e^{i \theta}$, $0<t<1$, determines an accessible boundary point of $G_{n}$. The function $v(w)$ has a radial limit at almost every point of $\partial D_{w}$.

If $E_{w}$ is the set of all points $e^{i \theta}$ in $\partial D_{w}$ satisfying the following conditions:

(1) the image of the radius $t e^{i \theta}, 0<t<1$, determines an accessible boundary point of $G_{n}$,

(2) $B_{n}(w)$ has a radial limit with modulus 1 at $e^{i \theta}$, and

(3) $v$ has a radial limit at $e^{i \theta}$, then $\partial D_{w}-E_{w}$ is of linear measure zero.

Let $e^{i \theta}$ be any point in $E_{w^{0}}$. If $e^{i \theta}$ corresponds to an accessible point @with complex coordinate in $\partial G_{n} \cap \partial D \cap\left\{z:\left|z-t_{0}\right|<3 r / 4\right\}$, and $P(@) \notin \tilde{E}$, $g_{n}^{-1}\left(A_{@}^{\prime}\right)$ (where $A_{@}^{\prime}$ is a suitable last part of $A_{@}$ contained in $G_{n}$ ) is an arc at $e^{i \theta}$ on which $\left|f\left(g_{n}(w)\right)\right|<m+\varepsilon$. At almost every such point $e^{i \theta}$ the radial 
limit of $v(w)$ is not greater than $\log (m+\varepsilon)$. For, otherwise, we would have

$$
\limsup _{w \rightarrow \partial D_{w}} v(w) \leqslant \log (m+\varepsilon) \text { on } g_{n}^{-1}\left(A_{@}^{\prime}\right)
$$

and

$$
\lim _{t \rightarrow 1} v\left(t e^{i \theta}\right)>\log (m+\varepsilon)
$$

thus $e^{i \theta}$ is an ambiguous point of the function $v(w)$. Hence there are only countably many such points by the Ambiguous Point Theorem (see [3] or [2, p. 85]).

If $e^{i \theta}$ corresponds to an accessible boundary point @ with complex coordinate $z(@)$ in $\left(\partial G_{n}-\partial G_{n} \cap \partial D\right) \cap\left\{z:\left|z-t_{0}\right|<3 r / 4\right\}$, then since $\phi(t)$ is continuous at $z(@)$ and $z(@)$ is a regular point of $\partial G$, we have

$$
\lim _{t \rightarrow 1} u\left(g_{n}\left(t e^{i \theta}\right)\right)=\phi(z(@))=\log 2 / m \text {. }
$$

Hence we obtain

$$
\limsup _{t \rightarrow 1} v\left(t e^{i \theta}\right) \leqslant \log 1-\log 2 / m<\log m .
$$

By Loewner's Lemma [1, p. 34], the set $\left\{e^{i \theta}: e^{i \theta} \in E_{w}, e^{i \theta}\right.$ corresponds to an accessible point@with $P(@) \in \tilde{E}\}$ is of linear measure zero. Hence altogether we have

$$
\limsup _{t \rightarrow 1} v\left(t e^{i \theta}\right) \leqslant \log (m+\varepsilon)
$$

for almost every $\theta$ in $0 \leqslant \theta \leqslant 2 \pi$.

Now let $v^{*}(w)$ be the harmonic conjugate of the harmonic function $v(w)$. Then the function

$$
F(w)=\exp \left(v(w)+i v^{*}(w)\right)
$$

is a bounded analytic function in $D_{w}$. Therefore it has radial limits almost everywhere on $\partial D_{w}$.

Let $C_{w}$ be the set of all points in $\partial D_{w}$ in which $F(w)$ has a radial limit whose modulus is not greater than $m+\varepsilon$. Then $\partial D_{w}-C_{w}$ is of linear measure zero.

Since $F(w)$ can be represented by a Poisson integral in terms of the values $F\left(e^{i \theta}\right)$ almost everywhere on $\partial D_{w}$, we have

$$
F(w)=\frac{1}{2 \pi} \int_{C_{w}} \frac{F\left(e^{i \theta}\right)\left(1-t^{2}\right) d \theta}{1+t^{2}-2 t \cos (\alpha-\theta)} .
$$

Thus

$$
|F(w)| \leqslant \frac{m+\varepsilon}{2 \pi} \int_{0}^{2 \pi} \frac{\left(1-t^{2}\right) d \theta}{1+t^{2}-2 t \cos (\alpha-\theta)}=m+\varepsilon
$$

that is, $e^{v(w)} \leqslant m+\varepsilon$ in $D_{w}$. Therefore $v(w) \leqslant \log (m+\varepsilon)$ for every $w \in$ $D_{w}$.

Let $w_{n}=g_{n}^{-1}\left(z_{n}\right)$. Then $v\left(w_{n}\right) \leqslant \log (m+\varepsilon)$, that is,

$$
\log \left|f\left(g_{n}\left(w_{n}\right)\right) / B_{n}(w)\right|-u\left(g_{n}\left(w_{n}\right)\right) \leqslant \log (m+\varepsilon) .
$$


Since $t_{0}$ is a regular boundary point of $G_{0}$, and $\phi(t)$ is continuous at $t_{0}$, we obtain

$$
\lim _{n \rightarrow \infty} u\left(g_{n}\left(w_{n}\right)\right)=\lim _{n \rightarrow \infty} u\left(z_{n}\right)=0
$$

Thus

$$
\limsup _{n \rightarrow \infty} \log \left|f\left(g_{n}\left(w_{n}\right)\right) / B_{n}(w)\right| \leqslant \log (m+\varepsilon) .
$$

Since $\left|B_{n}(w)\right|<1$, we have

$$
\limsup _{n \rightarrow \infty} \log \left|f\left(g_{n}\left(w_{n}\right)\right)\right| \leqslant \log (m+\varepsilon) .
$$

Therefore

$$
\limsup _{n \rightarrow \infty}\left|f\left(z_{n}\right)\right| \leqslant m+\varepsilon<m+(c-m) / 8<m+(c-m) / 2,
$$

contrary to our assumption.

Case 2. $m=0$. Choose an arbitrary $\varepsilon, 0<\varepsilon<1$, and replace $m$ by $\varepsilon$. Then by the result in Case 1, we have

$$
\lim _{z \rightarrow t_{0}, z \in D}|f(z)| \leqslant \varepsilon
$$

Since $\varepsilon$ was arbitrary, we have

$$
\lim _{z \rightarrow t_{0}, z \in D}|f(z)| \leqslant 0
$$

This completes the proof ot the Theorem.

As immediate consequences of the above Theorem we have the following corollaries.

COROllary 1. Let $D$ be a simply connected domain in the z-plane, which is not the whole plane, and $t_{0}$ a boundary point of $D, \tilde{E}$ a conformal null set of prime ends of $D$. If $f(z)$ is meromorphic in $D$ and bounded in the intersection of $D$ with some neighborhood $N\left(t_{0}\right)$ of $t_{0}$, and at each accessible boundarypoint@ withP(@) $\in \tilde{D}-\tilde{E}, z(@) \in \partial D \cap N\left(t_{0}\right)$, there existsanarcA@at $z(@)$ on which lim $\sup _{z \rightarrow z(@), z \in A @|f(z)| \leqslant m \text {, then }}$

$$
\lim _{z \rightarrow t_{0}, z \in D}|f(z)| \leqslant m
$$

COROllary 2. Let $D$ be a simply connected domain in the z-plane, which is not the whole plane, and let $t_{0}$ be a boundary point of $D, E$ a subset of $\partial D$ such that the set $\{P(@): z(@) \in E$, @ is an accessible boundary point of $D\}$ is a D-conformal null set. If $u(z)$ is harmonic in $D$ and bounded above in the intersection of $D$ with some neighborhood of $t_{0}$, then

$$
\lim _{z \rightarrow t_{0}, z \in D} u(z)=\lim _{z(@) \rightarrow t_{0}, z(@) \in \partial D-E}\left(\inf _{A_{@}}\left(\lim _{z \rightarrow z(@), z \in A_{0}} u(z)\right)\right),
$$

whereA@is an arc at an accessible boundarypoint@with $z(@) \in \partial D-E$ and the convergence is in the sense of the ordinary euclidean metric. 
COROLlaRY 3. If $E$ is of $\frac{1}{2}$-dimensional Hausdorff measure zero, or of logarithmic capacity zero, on the boundary of $D$ (in place of the assumption on $E$ in Corollary 2), then the same conclusion holds as in Corollary 2.

Note that $E$ is a conformal null set [4].

Corollary 4 (BAGEMihl [5]). Let $D$ be a simply connected domain which is not the whole plane and let $f(z)$ be an analytic function in $D$. Let $\tilde{E}$ be a set of prime ends which is mapped to a set of linear measure zero under a one-to-one conformal mapping of $D$ onto a unit disc $D_{w}=\{w:|w|<1\}$. Suppose that at every prime end $P$ of $D$ that does not belong to $E$ there is a curve $A_{P}$ such that $\lim \sup _{z \rightarrow P, z \in A_{P}}|f(z)| \leqslant m$ and that $f(z)$ does not have the asymptotic value $\infty$ at any prime end of $D$. Then it follows that $\sup _{z \in D}|f(z)| \leqslant m$.

\section{REFERENCES}

1. K. Noshiro, Cluster sets, Springer-Verlag, Berlin, 1960. MR 24 \# A3295.

2. E. F. Collingwood and A. J. Lohwater, The theory of cluster sets, Cambridge Univ. Press, New York, 1966. MR 38 \#325.

3. F. Bagemihl, Curvilinear cluster sets of arbitrary functions, Proc. Nat. Acad. Sci. U.S.A. 41 (1955), 379-382. MR 16, 1095.

4. K. Matsumoto, On some boundary problems in the theory of conformal mappings of Jordan domains, Nagoya Math. J. 24 (1964), 129-141. MR 34 \#324.

5. F. Bagemihl, A curvilinear extension of the maximum modulus principle, Proc. Nat. Acad. Sci. U.S.A. 63 (1969), 36-37. MR 41 \# 7100.

Department of Mathematics, University of Wisconsin, Milwaukee, Wisconsin 53201

Current address: Department of Mathematics, Inha University, Inchon, Korea 